\title{
Review Articis
}

\section{Acute Viral Encephalitis}

\author{
A R M Saifuddin Ekram ${ }^{1}$, Md. J Hossain ${ }^{2}$, Sultana M Hussain ${ }^{3}$, Md. M Alam ${ }^{3}$, S M A Hossain 4 , \\ I Ahmed ${ }^{5}$, Md. F Rahman ${ }^{6}$, S M K Nahar Begum ${ }^{7}$, E Gurley ${ }^{8}$, Robert F Breiman ${ }^{9}$
}

\begin{abstract}
Viral encephalitis remains a significant public health problem world wide including Bangladesh. Its transmission is either from insect or environmental vectors or from human-to-human contact. Corebrospinal fluid analysis, viral studies, and various imaging techniques provide sensitive and specific methods to detect many of these viral pathogens. Rapid diagnosis of encephallis allows early intervention in those conditions that are responsive to specific antiviral treatment, such as herpes simplex encephalitis. Specific treatment is available for certain viral encephalitides, but for others only supportive therapy is available. Vaccines are available to prevent a few important causes of encephalitis, but prevention of encephalitis revolves around minimizing exposure to pathogens, and insects which transmit many of them. Control must be based on rapid recognition of early cases, subsequent immunization of persons or animals at risk, or immunization of persons or animals with the potential to be at risk, such as traveiers, laboratory personnel, and attending clinicians.
\end{abstract}

TAd 2003; 16(1): 32-38

\section{Introduction}

Viral infections of the central nervous system (CNS) pose a threat to the health of peoplc throughout the world. Many viruses are both neurotropic and neuropathic. Diagnostic virology has advanced considerably during the past decade, allowing clinicians to use rapid, sensitive, and specific methods to detect many of these viral pathogens. However, effective treatment of CNS viral disease remains difficult because only a few pathogens can be treated with specific antiviral agents. ${ }^{\prime}$
We review current diagnostic and therapeutic strategies and advancements that can be used to improve the outcome for patients with viral encephalitis.

Encephalitis implies inflammation of the brain substance, parenchyma, which may coexist with inflammation of the meninges (meningoencephalitis) or spinal cord (encephalomyelitis) Encephalitis may be mild and self-limited, or may produce a devastating illness. ${ }^{2}$

\footnotetext{
'Associate Prolessor, Department of Meckine, Rajshahi Medical College Raishahi.

i Mecical Officer, Clinical Senences Division, ICDDR, B, Monakrial, Dhaka.

1 Mecical Oticer, IDU, HSID, ICDOR, B, Mohakhali. Dhakg.

i Lecuurer, Depariment of Microbioicgy, Rajshani Medical College, Rajshant.

¿ Prolessor, Department ot Microbialogy, Rajshahi Medical Colege, Rajshahi

6 Professor, Department ol Patholkgy. Rajshahi Medical Callege, Rajshahi.

1 Consultant Pathologist Xylia Mecicart, Rajshahi.

i Progremme Co-ordinator. Programme on Intectious Diseases and Vacoine Sciences. ICDDA, B, Mohalkhall, Dhaka.

F Head. Programme on Inlectious Diseases and Vaccine Scierices, ICDDA, B, Monakhali, Dhaka.
} 


\section{Epidemiology and causative organisms}

More than 100 different viruses can cause acute encephalitis. The likely pathogens in encephalitis are dramatically influenced by geographic location, traveling history, animal and insect exposures, and vaccination. Identifying the specific cause is key to effective management. ${ }^{3}$

Japanese encephalitis is a serious public health problem which has been shown to occur essentially wherever it has been studied in Asia including India ${ }^{4}$, Bangladesh (ICDDR, B, unpublished data), and in northern Thailand ${ }^{5}$. It is likely that JE virus is also an under-recognized cause of fever in other parts of Asia where infection with JE virus is endemic ${ }^{5}$. The incidence of Japanese encephalitis (JE) in Japan has dramatically decreased to a few patients per year since routine JE immunization was begun: however, JE remains a threat for those with decreased or absent immunity. ${ }^{6}$ Over the past five years, the distribution of Japanese B encephalitis (JE) virus has expanded into Australia via the Torres Strait Islands.

Herpes simplex virus type 1 (HSV-1) encephalitis comprises the majority of cases in Japan. It is also the most common cause of non-seasonal encephalitis in Australia while Murray Valley encephalitis (MVE) virus causes seasonal epidemics of encephalitis at times of high regional rainfall in Australia. ${ }^{8}$ This arthropod-borne virus is the most common flavivirus to cause encephalitis in Australia:

Other common causes in Australia are enteroviruses (including enterovirus type 71. which has recently caused epidemics of meningoencephaJitis) ${ }^{10}$, influenza virus and Mycoplasma pneumoniae. In addition to $\mathrm{JE}$ and MVE, two novel encephalitis viruses were recently identified in Australia - Hendra virus" and Australian bat lyssavirus. ${ }^{12}$ They should be considered if there is a history of animal exposure (horse or bat, respectively), or no other pathogen can be implicated.

The Centers for Disease Control and Prevention (CDC) reports as many as 1000 cases of primary encephalitis annually in the USA. However, the data likely underestimate the actual numbers of cases because specific pathogen is identified in only $50 \%$ to $75 \%$ of infected persons. ${ }^{13,14}$

In Bangladesh, the epidemiology and etiology of encephalitis are poorly defined. A recent ICDDR. $B$ report reveals that there were 4 outbreaks of encephalitis caused by Nipah/Hendra- like viruses and occurred in separate areas in Bangladesh in 2001,2003 and $2004^{15}$ All outbreaks occurred over brief periods and had high case fatality rates. In contrast to previous experience of Hendra and Nipah viruses in Australia, Malaysia and Singapore, in Bangladesh exposure to sick animals rarely has preceded illness; during a recent outbreak in Bangladesh person to person transmission was clearly shown. Fruit bats (Pteropus giganteus) have been the reservoir for the virus in all settings where epidemics have occurred. Systematic surveillance for encephalitis is not routinely done in Bangladesh. It is possible that other outbreaks and sporadic cases of Nipah/Hendra-like virus encephalitis have occurred or will occur. More information will be needed to define the magnitude of the problem and to identify strategies to prevent illness and deaths. A hospital based surveillance study is now being conducted in collaboration with ICDDR, B and 3 Medical College Hospitals in Rajshahi, Mymensingh and Dhaka to define the epidemiology and aetiology of encephalitis in Bangladesh."

Public health measures and vaccination have had a considerable impact in reducing the occurrence of viral encephalitis e.g. the measles-mumps-rubella (MMR) vaccine has eradicated mumps meningoencephalitis, measles encephalomyelitis, and subacute sclerosing panencephalitis (SSPE) in regions with compulsory immunization. ${ }^{16.17}$. Similarly, vaccination for the JE virus affords protection against JE encephalitis. ${ }^{18}$ Finally. human post-exposure prophylaxis and the quarantine and mandatory vaccination of pets have been effective in reducing the incidence of human rabies. ${ }^{19}$

Arthropod-borne viruses (arboviruses) and the rabies virus are transmitted by environmental vectors, whereas others, such as the herpesviruses, 
enteroviruses, and human immunodeficiency virus (HIV), are acquired by contact with infected humans. The distinction between environmental vector transmission and human-to-human transmission provides a useful paradigm for understanding the epidemiology of important viral pathogens.

\section{Environmental and lnsect Vectors}

Arboviruses have unique seasonal and geographic distributions that correspond to the habitat and life cycle of the vectors. California encephalitis (CE), from the LaCrosse strain, is the most commonly recognized arboviral infection in the US; it affects children between the ages of 5 and 15 years during July through September. ${ }^{20}$ Cases cluster in the central US, especially Ohio and Wisconsin, reflecting the habitat of the mosquito vector Aedes triseriatus.

Host characteristics or behaviors also influence the epidemiology of viral encephalitis. Persons who live near the insect habitat or engage in activities that facilitate contact with ticks or mosquitoes increase their risk. Tick-borne encephalitis of Europe, for example, occurs more commonly among foresters, shepherds. and hikers. ${ }^{21}$

\section{Human Transmission}

Viruses transmitted by humans occasionally display seasonal variations. Mumps virus, once the most common cause of meningoencephalitis. typically produced disease during the winter and spring. ${ }^{2}$ Likewise, nonpolio enterovirus infections, important causes of aseptic meningitis and potential causes of encephalitis, can cause local or widespread outbreaks in the summer and fall.

Minimal seasonal variation is found in encephalitis caused by members of the herpesvirus group, which includes herpes simplex virus (HSV), cytomegalovirus (CMV). Epstein-Barr virus (EBV), varicella-zoster virus (VZV), and human herpesvirus type 6 (HHV-6).

Intravenous (IV) drug use and certain sexual behaviors increase the risk of infection by HIV or herpesviruses. Additionally, persons with AIDS are at an increased risk of disease caused by several neuroinvasive viruses such as CMV, HSV, VZV, or the JE polyoma virus.'

\section{Emergence or reemergence of viruses}

Emergence or reemergence of viruses may be due to virus evolution, to the impact and influence of human populations on previously undisturbed ecosystems. We should understand the basic mechanisms by which these viruses emerge or reemerge and cause illnesses. Methods for detecting infections caused by yiruses and for detecting viruses or their genome sequences are available and improving. Methods for detecting antibodies also have improved.

Examples of recently recognized viruses causing encephalitis in humans, livestock, or wildlife include Hendra and Nipah viruses (benipaviruses: family Paramyxoviridae, genus Henipavirus) and Australian bat lyssavirus (family Rhabdoviridae. genus Lyssavirus). All three viruses are neurotropic and fruit bats (Pteropus spp.) are their natural hosts. Progress is being made in understanding transcription regulation and cell fusion by henipaviruses. ${ }^{23}$ This can help in future the development of vaceines against this pathogens. New encephalitis patterns reflect the roles that biologic reservoirs and vectors play in determining virus-human interactions. "New" viral encephalitis can also result from human host modifications that inerease susceptibility to neuroinvasive viral infection. Three human viruses, Nipah virus, Human Herpesvirus-6, and West Nile virus, present examples of how "new" viral encephalitides emerge in a specific geographic region or clinical setting. Nipati virus encephalitis emerged after the molecular evolution of a new zoonotic viral genus within the Paramyxoviridae family. Human herpesvirus-6 encephalitis has emerged in the immune suppressed human host harboring this ubiquitous but typically benign herpesvirus. West Nile virus encephalitis has emerged in the Western hemisphere after apparent abrupt translocation of this mosquito-borne virus to a distant geographic region with immunologically naive avian and human hosts. While the clinical features of these viral encephalitides are somewhat distinct, they each emerged as the result of human-derived factors that altered the biologic dynamic between humans and their viral pathogens. ${ }^{24}$ 


\section{Pathogenesis}

Experimental studies during the past several years have improved our understanding of how viruses infect the CNS. Viruses acquired from environmental vectors, such as mosquitoes or ticks, are inoculated directly through the host's skin. where the viruses replicate locally as well as in regional lymphoid tissues. Viruses acquired by human contact, such as the enteroviruses, attach to and penetrate mucosal surfaces and then replicate in regional lymphoid tissues. Next, a primary, lowlevel viremia distributes the virus systemically and allows the vinuses to replicate to high concentrations in extraneural tissues, A secondary viremia ensues, causing nonspecific symptoms such as fever, malaise, and headache. This additional wave of viremia may provide an opportunity for neurotropic viruses to breach the blood-brain barrier and infect neurons and other cells within the CNS. The precise mechanism by which viruses enter the CNS is not fully understood but may involve replication within vascular endothelium or direct transport of viruses across endothelial barriers by virus-infected Jymphocytes or macrophages. Another important mechanism of entry is retrograde axonal transport of viruses from peripheral sites directly to the CNS, as exemplified by rabies virus, HSV, and possibly the polioviruses. If 15 Once these pathogens invade the CNS, neurotropic viruses cause cellular injury by a combination of at least 3 mechanisms:

1. They may directly infect and destroy neurons or other CNS cells.

2. The immune response of the host may damage blood vessels or neural tissues by the development of virus-specific, cell-mediated immunity and the elaboration of inflammatory cytokines and neutralizing antibodies.

3. Finally, tissue edema, increased intracranial pressure, and altered cerebral blood flow induced by infection and inflammation may contribute to additional and potentially irreversible tissue damage. '

\section{Clinical manifestations}

Onset of encephalitis usually is acute, but signs and symptoms of CNS involvement often are preceded by a nonspecific, acute febrile illness. Presenting symptoms are headache and malaise, irritablity and lethargy. Fever, nausea. vomiting. neck pain, photophobia and hallucinations are common. Alteration of level of consciousness ranges from mild lethargy and confusion to coma. Generalized or focal neurologic abnormalities may be stationary, progressive, or fluctuating. Seizures are common, and unprovoked emotional bursts and loss of bowel and bladder control may occur. Clinical signs and symptoms of postinfectious encephalomyelitis may resemble those of acute viral encephalitis. ${ }^{b}$

\section{Diagnosis}

Patients with fever and signs of neurologic dysfunction require prompt diagnostic evaluation consisting of a cerebrospinal fluid (CSF) examination, electroencephalogram (EEG), and magnetic resonance imaging (MRD). The immediate goal in the evaluation of patients with possible viral encephalitis is to differentiate the clinical ieatures of viral encephalitis and nonviral disorders requiring antibiotics or antiinflammatory therapy. Virtually most of the patients coming with history of short duration of fever with altered consciouness and varied brain dysfunctions, are the cases of acute viral encephalitis if not proved otherwise. Examination of cerebrospinal fluid (CSF) in a patient with viral CNS infection reveals a normal or elevated opening pressure. A moderate elevation in white cell count (up to several hundred per cubic millimeter) is typical. Other typical CSF findings include a normal or elevated protein level and a normal glucose level. A routine Gram stain should also be obtained. In addition, CSF should be cultured for bacteria, viruses, fungi. and mycobacteria. Viral cultures of CSF are positive in $50 \%$ to $70 \%$ of patients with enteroviral meningitis but in a smaller percentage of those with encephalitis. Viral cultures from the nasopharynx and rectum are helpful in selected cases if the results are positive. In rare cases. arboviruses are isolated from blood during the acute phase of illness. 
Polymerase chain reaction ( $\mathrm{PCR}$ ) is being more widely used to detect viral DNA or RNA in CSF. This method has been reported to bave greater than $95 \%$ sensitivity and $100 \%$ specificity for HSV DNA in patients with biopsy-proven HSV encephalitis. PCR techniques show excellent specificity and sensitivity in the diagnosis of enteroviral meningitis, and use in diagnosis of encephalitis appears promising.

Magnetic resonance imaging (MRI) and computed tomography (CT) can provide useful information in the evaluation of encephalitis. MRI is the more sensitive test. Changes in acute infectious encephalitis include edema and abnormalities of the basal ganglia, cortex, and gray-white matter junction. An MRI scan may be normal early in the course of HSV encephalitis, but within days focal edema and hemorrhage usually are evident. Eastern equine encephalitis produces focal abnormalities involving the basal ganglia and thalami. MRI findings with other arboviral encephalitides are less well characterized.

Electroencephalography is less sensitive, but may be helpful if it shows characteristic features (eg, lateralising periodic sharp and slow wave patterns).

As diagnostic methods are evolving rapidly, it is important to contact the laboratory and provide sufficient concurrent sera and CSF for the optimum test protocol. In Bangladesh, the following tests at minimum should be performed in patients suspected to have encephalitis: CSF study, blood sugar, serum creatinine, serum electrolytes and liver function tests.

\section{Differential Diagnosis}

Various disorders can produce symptoms or signs that mimic those of encephalitis ${ }^{1.2}$ The major differential diagnosis is meningitis or parameningeal infection, especially if meningitis is caused by atypical pathogens such as Listeria monocyrogenes, $M$. tuberculosis or $C$. neoformans. Other causes of encephalopathy should be considered, including:

- Metabolic disturbances (hypoglycaemia, hyponatraemia and hypocalcaemia);
- Thiamine deficiency (Wernicke's enceptalopathy):

- Drugs (including neuroleptics [neuroleptic malignant syndrome], trimethoprimsulfamethoxazole, isoniazid, non-steroidal anti-inflammatory drugs, intoxications): and

- Inflammatory disorders, such as Behçet's disease, Reye's and Guillain-Barré syndromes. sarcoidosis, cerebral vasculitis (with or without a cerebral vascular accident). systemic Iupus erythematosus and Wegener's granulomatosis.

- Statas epilepticus and malignancy (paraneoplastic syndrome) may also sometimes be confused with encephalitis.

\section{Treatment}

Patients with encephalitis -

- Require hospitalization in a skilled nursing unit. Patients with viral encephalitis should be nursed in isolation to avoid the spread of infection and cross infection to other patients and other health care staff. Universal precautions must be practiced.

- Nursing care starts from the time the patient is admitted to the medical ward and the patients has to be observed intensely with regard to their general condition, complaints, and most importantly, all their neurological changes, vital signs, change of behaviout and any deterioration in their condition.

Above all, total patient care should be initiated to ensure comfort and to maintain hygiene at all times. Therefore, to maintain a constant standard of nursing care, a sufficjent number of nurses are needed.

Frequent assessments of the level of consciousness are required as well as careful monitoring for potential complications, such as seizures, increased intracranial pressure, or inappropriate antidiuretic hormone secretion.

Clinicians must individualize their management of patients with encephalitis according to the severity of the patient's condition and the availability of specific chemotherapy. 
Until a bacterial cause of CNS inflammation is excluded, parenteral antibiotics should be given. Treatment with a third-generation cephalosporin, such as cefotaxime sodium or cefuriaxone sodium, is recommended, Vancomycin should be added in geographic areas where strains of $S$ pmeumoniae resistant to penicillin and cephalosporins have been reported.

Empirical therapy with intratenous acyclovir should be initiated in HSV encephalitis. The recommended dosage is $30 \mathrm{mg} / \mathrm{kg}$ per day in three divided doses for 14 to 21 days. Relapse may occur. and retreatment should be considered.

With the recognition that anterial thrombosis may play an important role in the pathology of the disease anti-thrombotic agenis aspirin and pentoxyfyline has been used in these patients.

Ribavarin, a nucleoside analogue which has in vitro activity against Hendra can be used in these patients of Nipah/Hendra encephalitis.

\section{Supportive Care}

Many forms of viral encephalitis cannot be treated with specific antiviral medications: these include the arboviral diseases (CE. EEE. St. Louis encephalitis, JE, and Western equine encephalitis); rabies: EBV encephalitis: and enteroviral encephalitis. Therapy in these conditions consists of antipyretics, intravenous fluids, and anticonvulsants when necessary, as well as other supportive measures.

Seizures, a common complication in patients with viral encephalitis, can be treated with lorazepam, phenytoin, or phenobarbital using standard ageand weight-appropriate doses, Occasional patients with severe encephalitis and refraciory seizures may require intravenous barbiturate therapy. Increased intracranial pressure contributes to the morbidity and mortality of encephalitis and may require hyperventilation, therapy with mannitol or other osmotic diuretics, and careful management of fluid and electrolyte balance.

Although there is debate regarding the role of corticosteroids in patients with acute encephalitis, most patients tolerate these drugs for short periods. Some patients may benefit substantially from such therapy. Persons with acute disseminated encephalomyelitis, a condition that mimics the clinical manifestations of encephalitis but has distinet MRI features, may respond dramatically to a short, pulsed course of IV methylprednisolone or dexamethasone.

\section{Prognosis}

Patients with viral encephalitis have a variable prognosis that is dependent on the virulence of the pathogen and immunocompetence of the host. Persons with enteroviral encephalitis, $C E$, and Epstein-Barr virus encephalitis have low rates of morbidity and mortality, even though these diseases cannot be treated with specific antiviral drugs. By contrast, symptomatic humàn rabies virnally always results in death despite vigorous supportive ineasures.

Mortality in untreated HSV encephalitis averages $50 \%$ to $70 \%$, but treatment with acyclovir reduces mortality to only $10 \%$ or $20 \%$. Unfortunately. occasional patients with HSV encephalitis relapse despite appropriate management, as well as survivors, have high rates of seizures and permanent cognitive or behavioral sequelae in spite of acyclovir therapy. In northern Australia, it may be desirable to distinguish $\mathrm{MVE}$ from Japanese encephalitis clinically. Both conditions frequently affect the brainstem and basal ganglia, but MVE often involves the spinal cord, while Japanese encephalitis may produce striking meningeal signs, with or without thalamic involvement. Both have high mortality $(25 \%-$ $33 \%$ ) and rates of chronic sequelae in survivors ( $50 \%$ ). ${ }^{2}$ Mortality of Nipah encephalitis outbreak in Malaysia was $40 \%$ and denth was due to severe brainstem involvement. ${ }^{26}$

\section{Conclusion}

Rapid diagnosis and early treatment of viral encephalitis are vital to reduce mortality and neurologic sequelae. Specific treatment is available for cerlain viral encephalitides, but for others only supportive therapy is available. Control must be based on rapid recognition of early cases, subsequent immunization of persons 
or animals at risk, or immunization of persons or animals with the notential to be at risk. such as travelers, laboratory personnel, and attending clinicians.

\section{References}

1. Bale J F. Diagnosis and Management Of Viral Encephalitis. Infect Med 1998; 15(8):547-556.

2. Beaman $\mathrm{MH}$, Wesselingh S L. Acute communityacquired meningitis and encephalitis. Med I Aus.2002 176 (8): 389-396.

3. Gutierrez K M, Prober C G. Encephalitis: Identifying the specific cause is key to effective management. Post Grad Med. 1998:103 ( 3 ):102.

4. Sehgal A; Dutta AK Changing perspectives in Japanese encephalitis in India. Trop Doct $2003 ; 33(3): 131-4$

5. Watl $G_{1}$ Jongsaku K. Acule Undifferentiated Fever Caused By infection With Japanese Encephalitis Virus. Am J Trop Med Hyg.2003;68(6):704-706.

6. Shoji H. Azuma K, Nishimura Y. Fujimoto H. Sugita $Y$. Eizuru $Y$. Acute viral encephaitis; the recent progress. Intem Med. 2002; 41 (6):420-8.

7 Hanna JN, Ritchie SA, Phillips DA, et al, Japanese encephalitis in north Queensland 1998. Med J Aust 1999; 170: 533-536.

8. Russell RC, Dwyer DE. Arboviruses associated with human disease in Australia. Microbes Infect 2000; 2: 1693-1704.

9. Whilley RJ, Lakeman F. Herpes simplex infections of the central nervous system. Clin infect Dis 1995. 20: $414-420$.

10. McMinn P, Lindsay K, Perera $D$, et al. Phylógenetic analysis of enterovirus 71 strains isolated during linked epidemics in Malaysia, Singapore and Westem Australia. J Virol 2001: $75: 7732-7738$

11. Allworth A, Murray K. Morgan J. A human case of encephalitis due to a lyssavirus recently identified in fruit bats. Commun Dis Intell 1996: 20: 504 .

12. Paterson DL, Murray PK. McCormack JG. Zoonotic disease in Australia caused by a novel member of the paramyxoviridae, Clin Infect Dis 1998; 27 . $112-118$
13 Centers for Disease Control: Table 1: Notifiable diseases: Summary of reported cases, Uniled States, 1981-1990. MMWR 39!55, 1990

14. Centers for Disease Control: Arboviral diseaseUnited Stales, 1991. MMWR 41:545-548, 1992

15. Outbreaks of encephalitis due to Nipah/Hendra-like vifuses in western Bangladesh. HSB 2003:1 (5) -1-6

16. Kimberlin DW Plotkin SA. Vaccines for viral diseases with significant CNS manifestations, in Scheld WM, Whitley RJ, Durack DT (eds): Inlections of the Central Nervous System New York, Lippincolt-Raven Publishers, 1997; 983-1012.

17. Koskiniemi M. Rautonen is Lehtokoski-Lehtiniemi E. Epidemiology of encephalits in children: A 20year survey. Ann Neurol, 1991;29:492-497

18. Poland JD, Cropp CB, Craven AB, ef al: Evaluation of the potency and salety of inactivated Japanese encephalitis vaccine in US inhabitants. $J$ infect Dis.1990; 161:878-882.

19. Fishbein DB, Robinson LE: Current concepts. Rabies. N Engl J Med. 1993;329:1632-38.

20. Tsai $T$ : Arboviral infections in the United States. Infect Dis Glin North Am 5:73-102. 1991.

21. Kunz C. Tick-borne encephalitis in Europe, Aota Leiden 60(2):1-14. 1992.

22. Koskiniemi $M$, Rautonen d, Lehtokoski-Lehtiniemi $E$, et al: Epidemiology of encephalitis in children: $A$ 20-year survey. Ann Neurol 29:492-497, 1991

23. Calisher C H, Dodet B, Griffin D. Emergence and Control of Zoonotic Viral Encephalits. Emerg Infect Dis 9(8): $1029 \cdot 1030,2003$.

24. McCarthy M. Newer viral encephalitides. Neurolog 2003 Jul; $9(4) ; 189-99$.

25. Cassady KA Whilley RJ: Pathogenesis and pathophysiology of viral infections of the centcal nervous system, in Scheld WM, Whilley RJ, Durack DT (eds): Intections of the Central Nervous System. New York, Lippincolt-Raven Publisners, 1997. pp 7-22

26. C T Tan. The Nipas encephalitis outbreak in Malaysia. Abstract of Joint international CPD meeling of BAATM, Dhaka 2004; 13 .
All correspondance to. A R M Sailuddin Ekram Associale Prolessur Deoartment of Mecticine Rajishahi Medical College Alajshahi. 